\title{
Une métaphysique selon le poétique
}

Lecture de Dufrenne

Frédéric Jacquet

\section{CpenEdition}

Journals

Édition électronique

URL : http://journals.openedition.org/alter/602

DOI : $10.4000 /$ alter.602

ISSN : 2558-7927

Éditeur :

Association ALTER, Archives Husserl (CNRS-UMR 8547)

\section{Édition imprimée}

Date de publication : 31 décembre 2018

Pagination : 103-121

ISBN : 978-2-9550449-4-0

ISSN : 1249-8947

Référence électronique

Frédéric Jacquet, « Une métaphysique selon le poétique », Alter [En ligne], 26 | 2018, mis en ligne le 31 décembre 2019, consulté le 26 mars 2020. URL : http://journals.openedition.org/alter/602 ; DOI : https://doi.org/10.4000/alter.602 


\title{
UNE MÉTAPHYSIQUE SELON LE POÉTIQUE LECTURE DE DUFRENNE
}

\author{
Frédéric Jacquet
}

Nous voudrions montrer que la phénoménologie enveloppe l'exigence d'une métaphysique, et que la voie vers cette métaphysique consiste en une heuristique poétique. Dans ce cadre, la phénoménologie se voit définie de façon renouvelée - car son acte inaugural, l'épochè, se trouve lui-même ressaisi de façon novatrice en tant qu'elle engage la question du sentiment, de l'esthétique et du poétique. Mais précisons d'abord que la métaphysique est intégralement repensée, elle désigne désormais la théorie de la Nature dont l'être est irréductible à ses figures livrées au sein de l'expérience sans que ce dépassement en fasse l'autre $\mathrm{du}$ sensible. Il est plutôt question d'une transcendance interne au sensible, et c'est en quoi la phénoménologie conduit à la méta-physique qui décèle le sens d'être de la Nature. La métaphysique est donc une proto-physique, et cette acception de la métaphysique rompt avec ses déterminations historiques, puisqu'elle n'est plus régie par la logique dominée par la recherche de l'identité essentielle et de la raison suffisante notamment - de même qu'elle rompt avec l'onto-théologie. Il ne s'agit plus de reconduire la question de l'être à celle de l'étant au point de s'enquérir de l'étant suprême en tant que principe de tout ce qui est (ce qu'a su établir Heidegger dans sa destruction de la métaphysique), logique métaphysique qui conduit in fine à aborder l'être sur fond de néant (ce que montre Bergson), Dufrenne s'inscrivant ainsi dans le double sillage de Heidegger et de Bergson quant à sa caractérisation de la métaphysique demeurée dogmatique ${ }^{1}$. Le vocable de métaphysique se

\footnotetext{
${ }^{1}$ M. Dufrenne, Le Poétique, [1963], Paris, PUF, 1973, réédité avec l'essai « Pour une philosophie non théologique », p. 56. Dufrenne retrouve, en un mode phénoménologique singulier, la critique
} 
justifie cependant car il est question de la quête d'un absolu et, conséquemment, d'un dépassement de l'expérience. Les voies vers cette transcendance - esthétique, poétique - permettent alors la suspension des principes de la métaphysique historique, Dufrenne cheminant vers une "philosophie non théologique $»^{2}$. De ce point de vue, il se démarque alors de la philosophie heideggérienne de l'être qui, à sa manière, consacre la mort de l'homme puisque l'homme, sous la figure du Dasein, n'est que le "serviteur de l'Être $»^{3}$ ayant seul l'initiative de la manifestation. L'exigence est donc de tenir dans la corrélation et de penser selon elle. Reconnaître l'appartenance de l'homme au monde, à l'être originaire, n'implique nullement la réduction de l'homme à une simple efflorescence ou un médium dépossédé de toute initiative. Ainsi en estil de la position de Heidegger qui reconduit, à son insu, le motif ontothéologique sous la figure d'une hypostase de l'Être ${ }^{4}$, brisant ainsi l'exigence corrélationnelle. L'heuristique poétique évoquée permet au contraire de penser depuis l'intérieur de la corrélation pour la déborder sans la nier. Cette heuristique n'est pas exclusive d'autres voies vers un au-delà de l'expérience, notamment la voie que nous dirons phénoménologique, mais il y a cependant un privilège de la voie poétique de la métaphysique, qui est plus largement celui de l'esthétique ${ }^{5}$. On assiste donc à une double focalisation - de la phénoménologie vers l'esthétique et de l'esthétique vers le poétique. Comprendre ce privilège suppose de restituer la teneur du problème rencontré par la phénoménologie. Or la phénoménologie se présente - depuis Husserl - comme une élaboration de l'a priori corrélationnel entre le monde et ses modes subjectifs de donnée, mais cette interrogation implique son propre débordement vers une métaphysique qui se spécifie en tant que métaphysique de la naissance $^{6}$. Il s'impose en effet de penser l'émergence du sujet de la

\footnotetext{
bergsonienne de la métaphysique qui s'enquiert d'une «origine absolue aux frontières du néant ».

2 Voir l'essai tout juste cité « Pour une philosophie non théologique », Le Poétique, op. cit., p. 7-57.

${ }^{3}$ M. Dufrenne, Pour l'homme. Essai, Paris, PUF, 1968, p. 27.

${ }^{4}$ M. Dufrenne, «Pour une philosophie non théologique », Le Poétique, op. cit., p. 11.

${ }^{5}$ Nous nous permettons de renvoyer à nos ouvrages : Naître au monde. Essai sur la philosophie de Mikel Dufrenne, Milan, Mimesis, «L'œil et l'esprit », 2014 ; La Transpassibilité et l'événement. Essai sur la philosophie de Maldiney, Paris, Classiques Garnier, 2017, p. 453-546.

${ }^{6}$ Il demeure que Dufrenne ne parvient pas à élaborer une telle métaphysique de la naissance, et l'interrogation sur la naissance demeure largement inchoative dans son œuvre. De notre côté, nous tentons d'édifier une philosophie de la naissance: nous nous permettons de renvoyer à notre Métaphysique de la naissance, Louvain-La-Neuve, Peeters, Bibliothèque philosophique de Louvain, 2018. Il s'agit pour nous d'élaborer une philosophie qui situe la question de la naissance en son cœur : l'épochè doit prendre la figure d'une épochè natale, la phénoménologie de la naissance conduit alors à une métaphysique de la naissance, foyer d'une anthropologie-selon-lanaissance qui enveloppe une phénoménologie de l'enfance, une esthétique, une érotique, une éthique, et une herméneutique de l'existence effectuées selon le phénomène de la naissance.
} 
corrélation et, partant, de définir le sens de l'Être de telle sorte que la manifestation puisse surgir en lui. Il ne s'agit toutefois pas de s'enquérir des conditions de possibilité de la manifestation mais d'exclure ses conditions d'impossibilité, car, dans le cas contraire, il faudrait que le sujet de l'apparaître soit préfiguré dans les choses, si bien qu'elles seraient déjà de l'ordre de la manifestation subjective (déjà sujets, et non plus choses, selon une perspective qui ne respecterait pas la logique de l'apparaître). Or cette interrogation, et les percées dufrenniennes, se situent dans l'orbite d'une métaphysique phénoménologique, la transgression de la phénoménologie étant accomplie selon ses données propres. La question est pourtant celle de la possibilité d'une telle transgression. Comment le sujet fini peut-il dépasser sa condition pour saisir le monde sans que cette saisie ne soit la conquête d'une nouvelle position subjective sur lui ? La question s'aiguise encore puisque l'homme, sujet corrélationnel, semble doublement séparé du monde - par sa naissance, qui consacre la survenue de la finitude dans l'être infini, faisant qu'il est inscrit en lui sans se réduire à un simple fragment, et par le langage, qui emprisonne dans l'abstraction des mots et qui imprègne la perception. Cette aliénation verbale accuse notre séparation à l'égard du monde. Or le poétique est précisément ce qui assure la possibilité de dire le monde à la faveur d'une réconciliation ontologique avec lui, et la poésie est ce régime du discours qui déjoue l'aliénation verbale de l'intérieur de la parole et qui livre ainsi la ressource au philosophe pour dire le monde sans l'homme qui n'est autre que la Nature. Mais ces percées s'enracinent dans l'état poétique de l'homme, que Dufrenne décrit comme un transcendantal paradoxal puisqu'il est une condition subjective qui, loin de constituer l'expérience, donne accès à ce qui transcende l'expérience. Cette expérience poétique repose sur le sentiment ${ }^{7}$, et la métaphysique dufrennienne est conquise selon ce que j'appellerai une heuristique du sentiment. Le sentiment, non réductible à l'émotion ${ }^{8}$, engage une épreuve du poétique, c'est-à-dire du monde que charrie le poème, et qui reconduit chaque fois à la réalité primordiale sur le mode du pressentiment: le monde, et finalement la Nature, transparait alors dans les mondes qu'elle porte. Le concept de monde désigne le plus souvent, dans le corpus dufrennien, la réalité expérimentée dans la perception

\footnotetext{
${ }_{7}$ Nous n'aborderons pas, dans le présent contexte, les analyses de Renaud Barbaras sur la question du sentiment (Métaphysique du sentiment, Paris, Cerf, 2016), il faudrait y consacrer un autre travail ; surtout, nous poursuivons nos réflexions, d'ailleurs antérieures, consacrées à l'œuvre de Dufrenne, et recueillies notamment dans Naître au monde, op. cit. : la question du sentiment y est décisive.

${ }^{8}$ M. Dufrenne, Phénoménologie de l'expérience esthétique, [1953], tome 2, Paris, PUF, 1967, p. 514 sq: nous l'expliquerons.
} 
ordinaire ou encore les mondes esthétiques que l'œuvre d'art déploie. Par contraste, la Nature désigne la réalité telle qu'elle existe indépendamment de l'homme, elle est donc le monde sans l'homme, qui lui préexiste : elle est l'originaire en lequel toute chose s'inscrit. Or, l'heuristique poétique, accomplie selon le sentiment, engage une réduction cosmologique qui renverse le transcendantalisme sans l'inverser et donne accès à la Nature en elle-même. La réduction ne résorbe pas le monde dans l'immanence subjective - comme l'idéalisme transcendantal poussé à sa limite (se montrant ainsi non fidèle aux leçons de l'intentionnalité) - et ne dissout pas non plus le sujet dans le fond qui le porte - à la manière d'un naturalisme, position symétrique inverse de l'idéalisme. Nous commencerons par fixer la voie poétique en montrant l'aporie qu'elle conjure, pour ensuite décrire l'heuristique du sentiment qui en est la cheville ouvrière, avant de penser la Nature comme telle, cœur de la métaphysique poétique.

\section{Le poétique}

Il convient en effet d'envisager la difficulté de principe que rencontre le projet métaphysique. La question est de savoir comment l'homme peut accéder à la Nature sans l'homme, puisque la Nature qui se donne à lui n'est pas la Nature sans lui. Autant dire que l'homme doit pouvoir se démettre de lui-même, faire l'épochè de son humanité, non bien sûr en un sens moral, mais selon une acception anthropologique, afin qu'il puisse dépasser l'obstacle de sa condition pour saisir le monde sans condition. Tout se passe comme si l'homme devait faire l'épochè de la finitude qui le caractérise afin de saisir ce qui l'excède, c'est-à-dire afin de penser à l'impossible. D'une certaine façon, l'homme est appelé à mourir à lui-même pour naître au monde, c'està-dire pour se glisser en lui, n'être plus rien afin de l'accueillir sans que rien dès lors n'entrave cette appréhension ; ce qui suppose de conjurer la distance corrélationnelle. Mais abolir cette distance ne supprime-t-il pas du même coup toute conscience, toute expérience et donc toute possibilité de dire le monde? La cosmologie semble devenir impossible - par disparition du sujet capable de l'élaborer - au moment où elle devient possible - lorsque le sujet se supprime lui-même pour que le monde paraisse tel qu'en lui-même. Pour le dire autrement : comment sortir de soi pour voir le monde sans que ce voir ne le réduise déjà au statut de monde pour moi ? La métaphysique se trouve donc empêchée par un obstacle anthropologique dirimant qui l'appelle autant qu'il l'interdit. Le projet métaphysique est requis en vertu de la fini- 
tude humaine car, séparé du monde par la réalité de son existence, on ne peut qu'admettre la transcendance du monde sur son apparaître subjectif, celle d'une réalité dont la présence précède et excède toute donation. Mais, dès lors, le monde s'avère inaccessible en vertu de cette précession et ne paraît que selon le masque de son apparition subjective. Autrement dit, ce qu'exige la constitution de la métaphysique - de transcender la condition humaine -, qui en fixe la possibilité, est aussi ce qui en consacre l'impossibilité ${ }^{9}$, puisque toute expérience de la Nature est toujours celle de la Nature naturée par l'homme, c'est-à-dire du monde, et non de la Nature en elle-même, telle qu'elle existe indépendamment de ses manifestations à la conscience : toute expérience humaine de ce qui précède l'homme est donc vouée à l'impossible. Pourtant, Dufrenne montre qu'il y a une "intuition de la Nature », celle-ci se donnant en "filigrane ${ }^{10}$, de façon présomptive; ce qui revient à dire que la finitude trouve une ressource contre elle-même et qu'il y a une attestation de la puissance du fond pour le sujet qui en est séparé. Ce paradoxe réside tout entier dans le paradoxe de l'acte de notre naissance au sein du monde qui conjure l'impossibilité de la métaphysique. Naître, c'est se séparer du monde sans rompre avec lui, si bien que l'épochè de la finitude prend la forme d'une épochè de la naissance, de notre séparation avec le monde, mais celle-ci puise dans cette double dimension de la naissance, dans les attaches qu'elle préserve avec le monde qui peuvent tenir lieu d'une initiation au monde tel qu'il est indépendamment de nous à la faveur d'une immersion en lui, en une sorte de bain cosmologique. Il s'agit de plonger dans le monde sans s'abolir en lui afin que l'expérience se fasse expérience $d u$ monde. Il est temps de montrer que Dufrenne envisage deux voies dans sa trajectoire vers la métaphysique : la voie strictement phénoménologique et la voie esthétique qui se spécifie notamment en une esthétique picturale et en une esthétique poétique ${ }^{11}$, $1^{\prime}$ expérience poétique possédant une priorité qu'il nous faut comprendre. Comme l'écrit Dufrenne, la pensée franchit le seuil qui conduit à une philosophie de la Nature « sur les ailes incertaines et superbes de la poésie $»^{12}$.

- La voie phénoménologique s'inaugure par une phénoménologie de la perception, découvrant que toute donation s'enlève sur fond de

\footnotetext{
${ }^{9}$ Voir par exemple « A priori et philosophie de la Nature », Filosofia, 18, 1967, p. 726-727.

${ }^{10}$ M. Dufrenne, Le Poétique, op. cit., p. 235, p. 227.

${ }^{11}$ Dufrenne s'intéresse aussi à la musique, nous renvoyons de ce point de vue à L'oxil et l'oreille, Paris, Éditions Jean-Michel Place, 1991.

12 M. Dufrenne, «A priori et philosophie de la Nature », Filosofia, 18, 1967, p. 731. Précisons que dans Le Poétique, Dufrenne souligne que la science, dès lors qu'elle comprend une énergétique, fait signe vers la puissance de la Nature.
} 
monde, qui est l'horizon de tous les horizons. De ce point de vue, il est question d'une reprise et d'une radicalisation de la théorie husserlienne de la donation par esquisses : toute expérience est forcément partielle, mais cette partialité n'est pas la contrepartie de notre déficience anthropologique, elle est au contraire une exigence ontologique. Et la profondeur de la manifestation laisse transparaître la profondeur métaphysique du monde sans $\mathrm{l}^{\prime} \mathrm{homme}^{13}$. En sa transcendance intérieure à la phénoménalité, le fond perceptif fait donc signe vers le fond cosmique, qui n'est autre que la Nature comme puissance contenant la potentialité de figurations in-finies sans lesquelles la dynamique perceptive s'éteindrait faute d'une profondeur à parcourir. Nous n'emprunterons toutefois pas cette voie afin de nous appesantir sur la voie esthétique, que suppose par ailleurs la voie phénoménologique. La voie esthétique accomplit en effet un pas supplémentaire : « Non seulement la réflexion doit désormais faire abstraction de ce que la phénoménologie ou la science ont pu lui apprendre, mais la conscience doit en quelque sorte faire abstraction d'elle-même ». Il s'agit bien d'accomplir le saut vers le fond, celui qui engage l'interrogation métaphysique. La voie esthétique permet d'atteindre la Nature « en deçà de la conscience », de sortir de la corrélation intentionnelle, et d'accomplir le saut dans le transcendant situant au niveau de la corrélation ontologique qui subordonne l'homme, comme partie de la Nature, à la dynamique de son devenir. Or, la voie esthétique se déploie sous le signe du " sentiment de la Nature », que Dufrenne appelle aussi «sentiment du fond », et c'est lui qui « incite à poser la question du fond audelà de la phénoménologie du fondement $\gg^{14}$, $\mathrm{c}^{\prime}$ est-à-dire au-delà de la corrélation. La voie esthétique, picturale, pour commencer par elle, permet d'effectuer l'épochè de la finitude et de "pressentir » la puissance $\mathrm{du}$ fond ${ }^{15}$ à même l'expérience du monde que livre le tableau. Ce qui est capté n'est autre que la profondeur ontologique qui affleure au sein de l'expérience et que l'expérience esthétique donne à sentir. La métaphysique est en un sens impossible, puisqu' elle est le fait d'un « homme qui devrait - et ne peut - s'abolir comme homme pour parler de ce qui est avant l'homme », mais, ajoute Dufrenne, "peut-être suffit-il que cette origine soit pressentie et conjurée, comme elle l'est dans $l^{\prime}$ expérience esthétique ${ }^{16}$. L'expérience picturale nourrit parfois ce

\footnotetext{
${ }^{13}$ M. Dufrenne, Le Poétique, op. cit., p. 199-211.

${ }^{14}$ Ibid., p. 200, p. 203, p. 207.

${ }_{15}$ M. Dufrenne, Phénoménologie de l'expérience esthétique, tome 2, op. cit., p. 656. ; Jalons, " AvantPropos ", La Haye, Martinus Nijhoff, 1966, p. 24 ; Esthétique et philosophie, tome 2, Paris, Éditions Klincksieck, 1976, p. 16, p. 211.

${ }_{16}$ M. Dufrenne, Pour l'homme, op. cit., p. 172.
} 
pressentiment de la puissance de la Nature, et c'est aussi le cas lorsque la Nature n'est pas le sujet du tableau; ainsi, les " portraits hollandais nous défient par une insaisissable profondeur», et ce qui s'exprime alors à travers « les voix du silence, $c^{\prime}$ est une Nature encore muette qui se montre sans se nommer ${ }^{17}$. La puissance de la Nature se trouve donc figurée dans l'apparaître du tableau à condition qu'il ait luimême un air de nature. Cette naturalité de l'objet esthétique recouvre alors sa spontanéité, sa plénitude d'être, l'intensité de sa présence qui exprime la spontanéité propre à la Nature, la dynamique de son devenir et sa puissance expressive. Dès lors, l'objet esthétique, le tableau évoqué, fait paraître l'épaisseur et l'inépuisabilité des choses que perd leur objectivation. Ainsi, l'esthétique picturale réforme le regard au point que le spectateur des Sainte-Victoire de Cézanne, par exemple, verra la profondeur minérale et sauvage de cette montagne qui est une cristallisation de la puissance élémentaire de la Nature. L'expérience esthétique est la voie vers le monde dont elle fait paraître l'inépuisable puissance à la surface du sensible.

- Seconde focalisation - la voie poétique. L'heuristique poétique possède un privilège insigne par rapport à l'esthétique picturale, car c'est avec l'esthétique poétique que la finitude franchit de la façon la plus accomplie la barrière de la finitude, le poète permettant de conjurer l'obstacle anthropologique, la séparation corrélationnelle, sans l'abolir. Cette conjuration s'opère alors que la séparation est à son acmé, à savoir au niveau du langage qui nous sépare du monde par son arbitraire (que théorise Saussure) et par sa puissance d'abstraction (celle des mots, des concepts qui généralisent l'expérience), et c'est la poésie qui l'engage, car elle possède une puissance de dévoilement qui excède celle des autres arts. Le privilège de la poésie est toutefois présenté de façon très nuancée. Comme l'écrit Dufrenne, l'unité de l'homme et du monde s'atteste à la fois de façon « plus précaire et plus profonde $»^{18}$ dans la poésie qu'avec les autres arts. Indiquons d'abord que l'esthétique picturale donne de son côté à voir l'affinité du monde et du sujet dans toutes les dimensions de son corps ; car le tableau - la chair des couleurs et le jeu de leurs tensions - s'adresse au corps dans sa dynamique perceptive, motrice, davantage que la poésie. L'affinité du corps avec le monde paraît alors dans toute son amplitude. En outre, on peut considérer que la poésie n'atteste l'unité de l'homme et du monde, et la puissance du fond, que selon une certaine précarité, parce que les "images sont déjà "mises en mots", et [que] c'est avec le

\footnotetext{
17 M. Dufrenne, Le Poétique, op. cit., p. 243-244.

18 Ibid., p. 195.
} 
langage que s'inaugure la scission $\gg^{19}$. Comprendre le privilège heuristique de la poésie suppose donc de saisir au plus près en quoi le langage, dans l'élément duquel la poésie se déploie, peut constituer un obstacle à l'accomplissement de sa fonction dévoilante, signe de la précarité évoquée. Mais, en vérité, le langage réalise la scission natale, accusant la séparation sur le mode d'un évidement cosmique, sans inaugurer lui-même cette scission. On ne saurait par ailleurs - comme Ricœur le reproche à Dufrenne ${ }^{20}$ - indexer purement et simplement la parole sur l'expressivité de la Nature, voire même sur le désir qu'elle aurait de se révéler. La parole suppose que le monde est à dire, qu'il témoigne d'une expressivité primordiale, mais aussi que l'homme désire le dire, ce qui n'est compréhensible que si ce dire engage un accomplissement ontologique de l'homme lui-même. En tout cas, dans le langage ordinaire, l'homme existe dans la scission pleinement advenue, dans l'absence du monde puisque parler, c'est viser à vide comme on le sait depuis les analyses de Husserl. Mais cela même qui fait la précarité de la voie poétique est aussi ce qui marque sa puissance heuristique. Examinons la teneur exacte de cette précarité et le remède poétique à l'enfermement linguistique.

Dans le langage, l'homme n'est plus mêlé au monde comme avec la perception, et c'est là aussi ce que manifeste l'usage prosaïque des mots qui généralisent et mutilent le réel. De ce point de vue, Dufrenne insiste en outre sur l'arbitraire du signe par lequel le langage provoque ce que Bonnefoy, selon une indéniable proximité avec Dufrenne, appelle une "privation de réalité ». En vertu de l'arbitraire évoqué, il $\mathrm{n}^{\prime} \mathrm{y}$ a aucune " ressemblance entre la chose nommée et son signifiant phonétique ", si bien que, par exemple, cet aspect qu'a la nuit d'être sombre, "n'est pas reflété dans le mot nuit, qu'on peut même voir clair », comme remarquait Mallarmé21. En cela le langage rend l'homme étranger au monde (alors qu'il lui appartient). Pourtant le langage contient aussi le remède contre cet empire des signes, qui effacent le réel au profit d'une construction intellectuelle. D'abord, Dufrenne montre que les signes du langage ne sont arbitraires « que lorsque l'on considère le langage du dehors ", ce qui est d'ailleurs pour une part légitime. Cependant, dans l'épreuve vivante de la parole, dont s'enquiert le phénoménologue, le sens n'est pas extérieur aux mots et l'on découvre qu'il adhère à leur "matérialité charnelle». Or, c'est cette

\footnotetext{
${ }^{19}$ Idem. Voir aussi p. 169.

${ }^{20}$ P. Ricœur, « Le Poétique (1966) », Lectures 2. La contrée des philosophes, Paris, Point essais, 1999, p. 335-347.

${ }^{21}$ Y. Bonnefoy, Entretiens sur la poésie (1972-1990), Paris, Mercure de France, 1990, p. 315, p. 245246.
} 
«saveur que précisément la poésie donne à goûter » en suspendant l'usage conceptuel du mot. Mieux : il s'agit de la situation originaire de la parole que la poésie retrouve puisque si le sens n'était pas d'emblée immanent à la texture des mots, ceux-ci ne pourraient jamais signifier par eux-mêmes. Il faut donc reconnaître que les mots enveloppent une puissance expressive. Cette expressivité du langage est la condition de sa puissance de signifier, y compris en sa version conceptuelle qui pourtant s'en abstrait. Si les mots, les signes, étaient totalement désertés par le sens, jamais ils ne pourraient signifier, de sorte que la fonction gnosique de la parole est nécessairement seconde, dérivée, et ne se déleste jamais totalement de sa fonction originairement expressive ; dans le cas contraire, le signe serait hors-sens, matière inerte, et il se perdrait comme signe. Il en découle que nos mouvements, même les plus détachés, ne brisent jamais totalement nos attaches avec le monde, ils s'en nourrissent bien plutôt, y compris la pensée la plus formelle. Mais il faut être plus précis car la question est alors celle du mode de la présence du sens aux mots; ce qui conduit Dufrenne à sa théorie de l'expression. Le point décisif est que l'expression tient à la chair sonore du mot qui rayonne d'un sens, et Dufrenne parle alors de leur « signification naturelle ». L'épochè poétique repose d'abord sur une suspension de la syntaxe ordinaire et l'attention doit se porter sur les « mots primordiaux » (ciel, or, nuit, palme, amour, mer, etc.) qui mettent en présence de la chose même. Ce sont les mots de la poésie qui, par elle, retrouvent leur liberté, n'étant plus assujettis à l'intentionnalité de la phrase. Le poème est alors régi par une syntaxe nouvelle, une syntaxe poétique, qui enveloppe une dynamique dont les mots sont le ressort (ce que néglige Ricœur dans son objection à Dufrenne ${ }^{22}$ ). Il faut alors déceler une relation entre le mot et la chose qui consiste en une ressemblance se jouant à l'intérieur du sujet entre « ce que suscite en nous le mot et ce que susciterait la chose ». Or le mot est expressif « lorsqu'en sonnant il nous fait résonner comme nous résonnerions à l'objet ». Il y a donc ressemblance entre l'expression évoquée par le mot, naissant de leur musicalité, et ce que la présence de la chose éveille en nous et que recueille le sentiment, mais il faut alors admettre que les choses se trouvent qualifiées par cette expressivité, qu'elles possèdent en elles-mêmes la puissance ontologique d'éveiller le sentiment - tel sentiment singulier chez celui qui s'y rend disponible. Ainsi le mot « nuit » nous donne le « sentiment de sa présence ${ }^{23}$ et, comme l'écrit Bonnefoy, le «nom de la

\footnotetext{
22 En dépit des nuances de son analyse, les objections de Ricœur ne nous paraissent pas légitimes (« Le Poétique (1966) », Lectures 2, op. cit., p. 345-346).

${ }^{23}$ M. Dufrenne, Le Poétique, op. cit., p. 91.
} 
nuit, quel qu'il soit, s'emplit alors de la nuit $»^{24}$. L'on voit que cette compréhension de la compréhension poétique des mots engage une « phénoménologie du sentiment ${ }^{25}$ qui se situe par-delà la distinction de l'intelligence et de l'affectivité, et le sentiment nous initie au monde en son être pré-objectif synonyme de son être-poétique. Ce qui est exprimé n'est pas un sens déterminé, à la manière d'un concept mais, au contraire, le sens des mots primordiaux est surdéterminé, témoigne d'une " plénitude encore ambiguë ». Ainsi le mot "jour » évoque un phénomène astronomique, une division du calendrier, la lumière en un sens lui-même plurivoque enveloppant la clarté de l'entendement, la pureté du cœur, etc. De cette ambiguïté constitutive des mots, de leur polyvalence, dépend la richesse des images poétiques qui font ellesmêmes signe vers l'infinie profusion de la Nature. Cette plurivocité des mots, et donc des images, ne paraît toutefois que sous la condition d'adopter l'attitude esthétique: l'état poétique rend sensible à cette expressivité des mots et du monde qu'ils révèlent. L'état poétique est alors moins le fait de l'imagination - qui enveloppe une puissance d'évocation mais « suggère l'irréalité de l'évoqué »-, que celui du sentiment qui définit notre sensibilité à la polyvalence des mots, aux images qu'ils recèlent, et consiste en une véritable intelligence de l'affectivité saisissant l'expression des mots. Si le sens est tout entier immanent aux sons dans le poème, à la matérialité des mots, l'expression requiert la signification, c'est-à-dire que l'œuvre possède un sujet. La signification dont il est question tient alors largement à $l^{\prime}$ « évocation d'une image ${ }^{26}$ dont le sentiment éprouve le sens qui s'illimite en un monde. La richesse du sens évoque et révèle en même temps la puissance de la Nature, la donne à sentir tout en manifestant notre communion avec le monde sous les registres eux-mêmes plurivoques de l'expressivité. Venons-en au sentiment en tant que ressort de l'heuristique poétique. La percée métaphysique n'est concevable qu'autant que l'homme est capable d'un état esthétique, et d'un état poétique (dont Dufrenne emprunte la notion à Valéry ${ }^{27}$ pour lui donner un sens singulier), celui d'une réceptivité originaire à la puissance originaire du monde primordial, de la Nature (son être poétique) qui trouve un « répondant dans la structure transcendantale du sujet ${ }^{28}$. Cette

\footnotetext{
${ }^{24}$ Y. Bonnefoy, Entretiens sur la poésie, op. cit., p. 246

${ }^{25}$ M. Dufrenne, Phénoménologie de l'expérience esthétique, tome 2, op. cit., p. 577.

${ }^{26}$ M. Dufrenne, Le Poétique, op. cit., p. 90-91, p. 122.

27 P. Valéry, CEuvres, tome 1, Paris, Gallimard, «Bibliothèque de la Pléiade », Édition établie et annotée par J. Hytier, 1957, p. 1319, p. 1321.

${ }^{28}$ M. Dufrenne, La Notion d'a priori, Paris, PUF, 1959, p. 288.
} 
réceptivité n'est autre que le sentiment qui possède une fonction heuristique dans la métaphysique poétique élaborée par Dufrenne.

\section{Le sentiment}

Dès les premières pages de son livre, Le Poétique, Dufrenne souligne que les percées accomplies s'effectuent sous le signe du sentiment, dans l'optique d'ailleurs d'une radicalisation métaphysique des leçons du livre de 1953 intitulé Phénoménologie de l'expérience esthétique. Or, confier le dire philosophique au sentiment - à ce que Dufrenne appelle les « évidences du sentiment »-, contrairement à ce qui pourrait sembler, ne revient nullement à céder à $l^{\prime}$ « obscurantisme $»^{29}$. Le sentiment est en effet ce qui initie au monde: il est comme l'attestation, en l'homme, de son appartenance ontologique permettant de découvrir le sens d'être du monde. Il s'agit alors de penser selon le sentiment, d'élaborer une philosophie, œuvre de la raison, en puisant à la source vive du sentiment en tant qu'il nous relie au monde et nous parle de lui. Dufrenne l'écrit admirablement : "Car [la] perception, lorsqu'elle devient sentiment, est capable de pensée : une pensée poétique qui se laisse conduire par la musique des mots et qui ne réfléchit pas, mais qui assume la fonction fondamentale de la pensée : le dévoilement, et qui peut, bien entendu, nourrir une réflexion ultérieure $»^{30}$.

Suivant l'acquis de la Phénoménologie de l'expérience esthétique, précisons que le sentiment possède une "fonction noétique » qui excède tout sens objectif et témoigne d'une puissance de "révélation »" Ainsi, le sentiment n'est pas l'émotion, purement subjective, et cette distinction s'avère décisive pour penser la spécificité de la perception esthétique en laquelle le sentiment est à l'œuvre puisqu'il l'accomplit. Ainsi une émotion comme la peur n'est pas le sentiment de l'horrible, enveloppant une dimension du monde, de même que la gaieté n'est pas le sentiment du comique, mais la façon dont nous pénétrons dans le monde du comique. Ajoutons que la terreur et la pitié ne sont pas le sentiment du tragique, mais des « réactions qui tiennent à la façon dont nous nous engageons dans le monde du tragique en nous associant aux héros de la tragédie ». Dès lors, peur, gaieté, pitié sont des mouvements, des commencements d'action alors que le sentiment est de l'ordre de la connaissance. Les sentiments évoqués sont des « connais-

\footnotetext{
${ }^{29}$ Ibid., p. 61-62.

30 Ibid., p. 144.

${ }^{31}$ M. Dufrenne, Phénoménologie de l'expérience esthétique, tome 2, op. cit., p. 472 ; Le Poétique, op. cit., p. 174 ; Jalons, «Avant-propos », op. cit., p. 25.
} 
sances-éclairs »; et Dufrenne écrit de ce point de vue : "Ainsi le sentiment a une fonction noétique : il révèle un monde, alors que l'émotion commente un monde déjà donné, soit pour le transformer magiquement, comme dit M. Sartre - et l'émotion est alors dérégulation -, soit pour engager une action valable, comme dit M. Ricœur ${ }^{32}$. L'expérience esthétique dévoile un monde singulier - comme le tragique singulier qui émane d'un tableau de Rouault - et j'éprouve alors la misère du monde sans éprouver l'angoisse ou la crainte qui, dans le monde réel, amorcent une action. Le sentiment est donc un pouvoir d'accueil, sensibilité à un certain monde si bien qu'il témoigne effectivement d'une puissance noétique, captant l'expressivité des choses qui est d'abord celle des œuvres. Mais le sentiment possède des significations stratifiées, puisqu'il est présenté dans L'inventaire des a priori comme l'ouverture première au monde qui en accueille l'afflux originaire, et c'est sur fond du sentiment cosmologique que la perception se déploie : "L'être-là du monde comme extériorité spatio-temporelle, $c^{\prime}$ est sans doute le premier a priori $»$. Or c'est le sentiment qui accueille cet être-là du monde ; Dufrenne l'indique : "Nous avons précisément inscrit parmi les a priori subjectifs le sentiment du réel; ce sentiment n'est pas une idée, l'idée que la science se fera du réel. C'est pourquoi il est aussi bien le sentiment du sur-réel, de cette puissance du réel qui se concentre en certains objets... $\gg^{33}$ Pour que la moindre perception ait lieu, et que le sujet corrélationnel aspire au monde dont il est séparé, il faut que le monde soit originairement donné, et c'est le sentiment qui assure la fonction de cette réceptivité originaire. Le monde est donné comme ce à quoi j'appartiens et dont je suis pourtant séparé, ce paradoxe renvoyant à celui de notre naissance. En effet, l'événement de la naissance est celui de la séparation à l'égard du monde, et d'abord à l'égard du corps de la mère, mais notre naissance préserve un lien avec le monde dont nous sommes séparés, si bien qu'il se manifeste selon cette distance native. Le désir - qui n'est autre que la dynamique de la perception - est alors cette tension vers le monde corrélative de la séparation native, qui suppose pourtant l'épreuve de ce bain cosmologique qu'engage notre appartenance et que le sentiment effectue. Mais le sentiment prend d'abord, dans les œuvres dufrenniennes, la figure du sentiment esthétique envisagé : il consiste dans l'intelligence de l'expressivité de l'objet esthétique, et il permet l'accueil du monde sin-

\footnotetext{
32 Ibid., p. 471-472.

${ }_{33}$ M. Dufrenne, L'inventaire des a priori. Recherche de l'originaire, Paris, Christian Bourgois Éditeur, 1981, p. 266, p 271. Nous l'avons établi dans les ouvrages précédemment cités sur Dufrenne et Maldiney.
} 
gulier exprimé par lui, comme le comique de Molière, irréductible à celui de Marivaux par exemple. Montrons dès lors que le sentiment esthétique reconduit au sentiment fondamental de la Nature car c'est elle qui, in fine, transparaît au sein des mondes esthétiques déployés.

Revenons de ce point de vue à la puissance de dévoilement du sentiment esthétique en nous focalisant sur l'état poétique. Or l'essentiel, de ce point de vue, est que le symbolisme poétique échappe à la logique rationaliste, inféodée aux principes de raison et d'identité, comme à son inscription au régime de l'émotion subjective, car il y a une "vérité du discours esthétique » ${ }^{34}$. On a montré que l'émotion de la gaieté n'est pas le sentiment du comique, et que chaque fois le sentiment fait paraître un monde singulier. Le sentiment est donc de l'ordre de la «connaissance » car il consiste en un dévoilement et la poésie, appelant l'éveil du sentiment, «n'explique pas, elle révèle » ${ }^{35}$. La magie de la poésie consiste alors à faire paraître la chose par la seule puissance de profération des mots ${ }^{36}$. Il y a une ressemblance entre ce que suscitent en nous les mots et le sentiment que susciterait la chose. Le sentiment est donc à " mi-chemin entre l'intelligence et l'affectivité »; et Dufrenne de préciser : " [Le sentiment met d']intelligence avec le contenu expressif du mot; mais cette intelligence n'est pas conceptuelle, elle est sensible ; et la sensibilité qui recueille le sens n'est pas sensorielle, mais affective ; nous communions avec ce qui nous est communiqué comme nous communiquons dans l'amour». Le sens capté n'est autre que le "visage affectif que l'objet tend vers nous $»^{37}$ qui irradie d'un monde et se gonfle à hauteur de monde pour finalement laisser pressentir la Nature - le sentiment étant, en son fond, c'està-dire en sa profondeur constitutive, sentiment du fond en sa profondeur infinie. C'est le sentiment même qui comprend la cohérence des images poétiques, celles-ci ne dépendant pas d'une logique conceptuelle mais des «synesthésies affectives ${ }^{38}$ qui disent le monde en exprimant des mondes singuliers selon le processus de percée cosmologique conduisant à déceler l'être poétique de la Nature. La Nature-fond est débordement, et elle se donne comme tel, c'est-à-dire selon la profusion des images. Chacune de ces images engage la profondeur d'un monde qui témoigne, à sa manière, de la profusion de la Nature, et la multiplicité des images exprime elle-même sa puissance expressive

\footnotetext{
${ }^{34}$ M. Dufrenne, Le Poétique, op. cit., p. 127.

${ }^{35}$ M. Dufrenne, Phénoménologie de l'expérience esthétique, tome 2, op. cit., p. 471 ; Le Poétique, op. cit., p. 126-128.

${ }_{36}$ M. Dufrenne, Le Poétique, op. cit., p. 137, p. 237.

37 Ibid., p. 92-93.

38 Ibid., p. 126.
} 
dans son caractère inépuisable. Ainsi, le sentiment est toujours en dernière instance sentiment de la Nature ${ }^{39}$ car le sentiment esthétique laisse pressentir sa puissance propre si bien qu'il enveloppe une dimension ontologique. Constituée selon le poétique, la métaphysique suit donc l'aiguillon du sentiment qui laisse pressentir sa puissance d'apparaître et en donne le pressentiment qu'il revient au philosophe de penser. Ce pressentiment est bien de l'ordre du sentiment, il est le statut que prend le sentiment en tant qu'épreuve de l'illimitation poétique qui exprime l'inépuisable Nature. Le poétique désigne donc l'expressivité des images que les mots enveloppent et qui dévoilent la puissance expressive de la Nature ; expressivité se donnant au sein du sentiment qui accomplit la perception esthétique, comme la perception des choses de la Naturedont la perception esthétique est la figure épurée. Autant dire que le sentiment, co-présent à toute donation, n'est autre que l'épreuve de notre affinité avec le monde (qui nous parle selon une expressivité primordiale) et celle de sa puissance inépuisable. Toute la démarche de Dufrenne consiste alors à montrer que le langage poétique recueille l'expressivité du monde, se déploie sous l'égide du sentiment, et que le philosophe peut alors hausser au langage conceptuel ces expériences privilégiées, élaborant une métaphysique phénoménologique. Le sentiment $\mathrm{du}$ fond, auquel le poète est particulièrement réceptif, est celui d'une puissance qui sous-tend l'apparition des choses et, comme l'écrit Dufrenne en 1959, «c'est d'abord au sentiment de la [N]ature » qu'il faut demander la vérité sur la Nature. Et Dufrenne d'ajouter: "[Le sentiment qu'exalte la poésie] accomplit spontanément la réduction phénoménologique, ou la remontée de l'étant à l'être $»^{40}$, c'est-à-dire vers la Nature comme sens ultime de l'être au point que la réduction poétique consiste en une réduction cosmologique. Il arrive à Dufrenne de parler de "sentiment océanique $»^{41}$ pour dire l'épreuve de ce qui est sans limite, infini, et qui coïncide avec le sentiment de la Nature. Élaborée dans un autre contexte par Romain Rolland, et interrogée par Freud, le sentiment océanique perd sa dimension religieuse pour désigner le sentiment naissant de l'immersion dans la Nature et par la participation à sa dynamique. L'éveil du sentiment en son amplitude océanique n'est autre que l'afflux originaire du monde qui livre le monde en son infinité constitutive. Le sentiment ne possède pas seulement la fonction théorique de voie vers la métaphysique, il est le fond de toute

\footnotetext{
39 « Nous appelons sentiment ce mouvement par quoi la conscience découvre à nouveau l'unité originaire dont elle émerge et pressent par là la Nature : tout sentiment est en quelque sorte sentiment de la Nature. » (L'inventaire des a priori, op. cit., p. 295).

${ }^{40}$ M. Dufrenne, La Notion d'a priori, op. cit., p. 288-289.

${ }^{41}$ M. Dufrenne, «L'imaginaire», Esthétique et philosophie, tome 2, op. cit., p. 116.
} 
perception, et il possède enfin une fonction existentielle d'ipséisation sous l'effet de l'exaltation selon lequel il se donne quand il passe au premier plan, le sujet ne se trouvant qu'en se perdant. Tel est ce qui se produit dans les expériences esthétique et amoureuse ${ }^{42}$. L'heuristique du sentiment est en tout cas la voie vers la métaphysique, et il importe alors de revenir sur la fonction dévoilante de la poésie afin de montrer que le chemin tracé ne cède pas à une compréhension restrictive de la poésie elle-même, et que, chaque fois, il y va d'une connaissance $d u$ monde au sens où elle le dévoile au sein du sentiment qui possède son régime spécifique d'intelligibilité, et reconduit finalement à la Nature en sa puissance propre.

\section{La Nature}

Une fois la fonction métaphysique, heuristique, du sentiment décelée, dès lors entendu comme puissance de dévoiler l'être du monde, ou mieux, de la Nature, une interrogation demeure : penser que la poésie dit le monde ne revient-il pas à céder à une compréhension de la poésie qui néglige la pluralité des «sujets » qui sont les siens ? Dufrenne répond très exactement à cette interrogation; d'une part, en considérant les différents types de sujets qui peuvent être ceux du poète, tout en montrant, in fine, que c'est toujours du monde dont il question ; d'autre part, les considérations sur le poétique dans le poète peuvent être lues comme une autre manière de justifier cette puissance métaphysique de la poésie ; nous laissons toutefois de côté ce second pan de l'analyse ${ }^{43}$. Cette heuristique poétique permet en tout cas d'élaborer une métaphysique de la Nature ${ }^{44}$ qui appelle un renouvellement de la compréhension philosophique de l'Infini.

Il convient d'abord de préciser que la poésie présente trois sujets possibles, qui se ramènent chaque fois au monde sans pourtant dissoudre du même coup leur sujet premier. Ces sujets sont la poésie ellemême, le poète, et le monde.

- Lorsque la poésie se présente comme "poésie de la poésie », de façon réflexive, elle ne se donne pourtant pas comme une réflexion attachée à un travail de conceptualisation, et ce type de poésie évoque un monde par la puissance charnelle des mots - voilà le point essentiel : « la réflexion poétique fait du monde de la poésie une poésie du

\footnotetext{
42 M. Dufrenne, Le Poétique, op. cit., p. 48. Nous l'avons établi dans nos ouvrages sur Dufrenne et Maldiney, notamment dans Naître au monde, op. cit., p. 231-246.

${ }^{43}$ Voir notre ouvrage, La Transpassibilité et l'événement, op. cit., p. 461 sq.

${ }^{44}$ M. Dufrenne, Art et politique, Paris, 10/18, 1974, p. 188.
} 
monde ; elle évoque un monde où la poésie a place comme une force de nature, albatros, bateau ivre ou cygne ». À la différence de la philosophie, la poésie réflexive ne s'enquiert pas d'un transcendantal poétique en quête de ses conditions et de ses potentialités, mais elle montre la poésie à l'œuvre dans un poème chaque fois singulier, c'est-à-dire qu'elle montre "comment la poésie est, et elle est d'abord dans le monde $»^{45}$.

- Ensuite, le poète peut écrire pour se dire lui-même en première personne, mais alors il exprime encore sa présence au monde, au monde singulier qui est le sien; celui de ses amours par exemple ; et si «l'œuvre est belle, elle prend une valeur exemplaire», l'universel paraissant alors sur la pointe du singulier. Chanter l'amour, c'est se soustraire au monde de l'utile et s'ouvrir à un monde singulier puisque « l'amour à travers l'aimée vise le monde comme décor, et peut-être aussi comme source ; car c'est du fond qu'il semble monter en l'amant qui l'éprouve comme un destin, et sa profondeur semble à la mesure de ce fond $»^{46}$. On pourrait indiquer la puissance heuristique de l'amour, mais il est dès lors clair que chanter l'amour, c'est aussi et, de ce fait même, chanter le monde qui brille à travers lui et lui donne son irremplaçable aura.

- Enfin, si le monde apparaît comme le vrai sujet de la poésie, il faut quand même dire que le poème porte sur une chose du monde (ou un amour, nous venons de l'indiquer) - lac, charogne, lessiveuse, etc. Certaines de ces choses sont poétisables à mesure de la puissance de manifestation dont elles témoignent, et appellent le dire poétique en cela qu'elles s'illimitent en un monde qu'elles cristallisent: elles portent ainsi $l^{\prime}$ «aurore [d'un] monde ». Cette puissance des choses trouve un écho dans la " force du mot», de certains mots (jour, palme, or, etc.), qui enveloppent une multiplicité de significations, jamais épuisées et qui, en raison de cette polyvalence, "évoquent un monde », en explore l'amplitude ou la polyvalence ontologique. Pour dire le monde, la poésie «joue avec ces mots à longue portée, puissants comme les phares dont l'éclat creuse l'espace $\gg^{47}$. Le poète creuse l'espace en ouvrant un monde, ce qui revient pour lui à dire le monde, à dévoiler la texture plurielle et pourtant une du sensible. Les images poétiques témoignent d'un sens immanent à la chair sonore des mots qui, libérés de la syntaxe grammaticale ou de la ponctuation ordinaire

\footnotetext{
${ }^{45}$ M. Dufrenne, Le Poétique, op. cit., p. 128-129.

${ }^{46} \mathrm{Ibid}$., p. 128-130. "Si Ronsard nous raconte ses amours, il est amoureux dans le monde de l'amour, mais il révèle aussi ce monde [...]. » (Ibid., p. 151). Voir aussi La Notion d'a priori, op. cit., p. 290.

${ }_{47}$ Ibid., p. 133-134.
} 
(de la prose ayant une visée intentionnelle), dévoilent le monde primordial, le font paraître en son expressivité. Mais, sans être poète, ajoute Dufrenne, $l^{\prime}$ « homme qui aime reconnaît l'amour en lui pour l'avoir connu dans les choses ; il dit qu'il brûle parce que la flamme est l'amour comme le chêne est la majesté et la souillure l'impureté, parce que les choses sont toujours signifiantes ». Ce faisant, il n'est pas question de céder à un anthropomorphisme naïf, mais bien au contraire de découvrir les vertus d'un " cosmomorphisme $»^{48}$. C'est en cela que la "poésie dit le monde », car le monde irradie d'un sens, d'une expressivité primordiale que le poète recueille pour la donner à voir au sein de ses poèmes, et cette expressivité est en vérité manifeste à tout homme qui se laisse investir par elle.

L'heuristique poétique ouvre donc la voie à une « intuition métaphysique $»^{49}$ qui donne accès à la Nature dans son infinie expressivité. On découvre ainsi une nouvelle figure de l'infini, qui n'est plus celle de Dieu, ni celle d'Autrui (Levinas), mais celle de la Nature en tant qu'infini apparitionnel ou infini poétique et expressif ${ }^{50}$. La Nature est pensée comme fond-puissance, elle est comprise comme un devenir, comme une dynamique qui enveloppe une puissance expressive, et, indique Dufrenne, la « vie est sans doute le témoignage le plus direct de la puissance à l'œuvre de la Nature ». Cette question de la vie fait signe vers la Nature comme devenir, comme mouvement de formation ou de constitution des choses, et cette notion de vie renvoie à la naturalité de la Nature. Cette naturalité n'est autre que sa plénitude, sa spontanéité et sa force d'apparaître, et c'est en cela que «la vie manifeste la poésie de la Nature ${ }^{51}$. Cette dynamique est toutefois fondamentalement expressive, et c' est pourquoi la Nature est décrite comme un « océan d'images » ou comme la «mère des images » ${ }^{52}$. L'image de l'océan connote l'idée d'infini, elle en est une approximation, et l'image de la mère fait signe vers la notion de foyer, de matrice, si bien que la Nature est décrite comme une puissance in-finie ou comme un infini d'expressivité : «Elle est la puissance de l'apparaître manifestée dans des images irrécusables et lourdes d'un monde ${ }^{53}$. L'essence de la Nature consiste dans sa dynamique apparitionnelle qui prend la figure $d^{\prime}$ 'une dynamique imageante : certaines images rayonnent $d^{\prime}$ un monde et toutes les images en leur caractère illimité expriment l'in-fini expres-

\footnotetext{
${ }^{48}$ Ibid., p. 130, p. 137.

${ }^{49} \mathrm{Ibid} ., \mathrm{p} .227$.

${ }^{50}$ M. Dufrenne, Jalons, « Avant-Propos », op. cit., p. 14.

${ }^{51}$ M. Dufrenne, Le Poétique, op. cit., p. 231, p. 192.

52 Ibid., p. 192, p. 234.

53 Ibid., p. 236.
} 
sif de la Nature. La Nature est donc irréductible à un sens déterminé car aucun sens n'en épuise la puissance mais elle est de l'ordre d'un sens incarné et rayonnant, d'une puissance inépuisable d'apparaître. Aussi la Nature ne se donne qu'en filigrane, et transparaît au sein du sentiment qui est la modalité adéquate de la saisie de son infinité. Il n'est dès lors plus question d'un (faux) infini prenant la figure d'une transcendance positive, celle d'un Dieu créateur irréductible au monde qu'il crée. L'infini n'est autre que la Nature en sa transcendance productrice et expressive, il s'agit d'un infini d'immanence sans pourtant que cette immanence naturelle ne résorbe toute transcendance. La puissance apparitionnelle de la Nature possède bel et bien une transcendance car rien ne l'épuise. Il est donc question de la transcendance d'une matrice inépuisable qui contient tout, y compris le possible qui s'invente et témoigne de l'expressivité de la Nature. Ce nouvel infini est donc également irréductible à l'infini de l'Autre tel que le décrit Levinas car l'Infini poétique est d'ordre ontologique alors que l'éthique lévinassienne engage la destitution de l'ontologie comme philosophie première. Au contraire, l'heuristique poétique creuse le sens de l'ontologie en dépassant l'ontologie du sensible vers une métaphysique de la Nature qui en révèle le sens d'être. Le réel est cependant pensé d'une façon toute distincte de la manière dont l'appréhende la métaphysique historique régie par le principe d'identité et de raison suffisante. Le philosophe pense désormais la Nature comme identité d'elle-même et de sa propre manifestation, ou de sa propre multiplication in-finie, synonyme de la puissance de la Nature dont la compréhension suppose de rompre avec le clivage du Même et de l'Autre. Cette métaphysique de la Nature est une philosophie non théologique qui n'aborde plus l'être sur fond de néant, et la Nature recouvre un sens renouvelé de l'éternité ${ }^{4}$, une éternité apparitionnelle irréductible à l'éternité définissant les idéalités comme à l'éternité religieuse, qui est hors-temps, et échappe au devenir. La Nature est cette puissance qui se confond avec ses apparitions spatio-temporelles même si aucune ne l'épuise, et qu'elles expriment ainsi le fond en sa dynamique expressive. Dans l'état poétique, les hommes pressentent cette puissance, et le poète cristallise, dans les images, l'expressivité de la Nature en la donnant à sentir. Le poétique est le miroitement de l'éternel dans le singulier manifestant l'être poétique de la Nature.

La démarche archéologique de Dufrenne découvre des figurations distinctes du réel, un réel stratifié ${ }^{55}$. Le réel n'est autre que le monde

\footnotetext{
${ }^{54} \mathrm{Ibid} .$, p. 216.

${ }_{55}^{5}$ M. Dufrenne, « Peindre, toujours », Esthétique et philosophie, tome 2, op. cit., p. 211.
} 
quotidien qui est façonné par l'intelligence et le langage, au point que la perception est pénétrée par leurs exigences, comme par celles de l'action. Cependant, ces constructions théoriques et pratiques supposent la première manifestation de la Nature, et c'est la perception sauvage qui consacre notre rencontre avec le réel, avec le sensible à l'état brut rayonnant d'une expressivité primordiale qui laisse pressentir la puissance de la Nature ou, mieux, la Nature comme puissance expressive que nomme désormais le concept d'Infini poétique. Cette métaphysique décèle la possibilité, pour l'homme, de se situer en deçà du tournant de l'expérience, pour accéder à l'absolu, dans le sillage revendiqué de Bergson, mais il s'agit désormais d'une métaphysique selon le poétique, que Dufrenne présente aussi comme un saut dans le monde sans l'homme, vers la Nature. Il rompt ainsi avec l'interdit kantien, et la poésie se donne comme une expérience de la transcendance (de l'inconnaissable selon Kant) ${ }^{56}$. Le rapport de la métaphysique à l'expérience, sous la figure de l'intuition évoquée, est donc distinct du rapport qu'ont les sciences à l'égard de l'expérience. C'est la notion de sentiment (et de pressentiment) qui s'avère de ce point de vue décisive par contraste avec le processus d'expérimentation - d'ailleurs pluriel - propre aux sciences. Dufrenne subvertit la distinction kantienne entre penser et connaître, puisque la pensée, visant le transcendant et s'élevant depuis le poétique, possède une valeur théorique, fonde la métaphysique d'un point de vue spéculatif du fait de cette intuition qui laisse pressentir la puissance de la Nature. Cette puissance apparitionnelle prend la figure d'un infini poétique que le poète donne à pressentir en jouant le langage contre lui-même. Le poétique n'est autre que cette puissance de la manifestation qui s'exprime dans les images, et qui infuse le niveau du langage, si bien que l'heuristique poétique dévoile l'affinité de l'homme et du monde, et la puissance infinie de la Nature elle-même. Elle se trouve donc atteinte en vertu de cette affinité ontologique et ne se donne pourtant que dans l'excès, dans la surabondance ou dans le débordement, mais cet excès est l'unique mode de manifestation de son infinité, livrant un sens inédit de la métaphysique qui pense la Nature comme Infini poétique.

${ }^{56}$ M. Dufrenne, L'inventaire des a priori, op. cit., p. 12. 